CHRONIC OBSTRUCTIVE PULMONARY DISEASE

\title{
Hyperglycaemia is associated with poor outcomes in patients admitted to hospital with acute exacerbations of chronic obstructive pulmonary disease
}

\author{
E H Baker, C H Janaway, B J Philips, A L Brennan, D L Baines, D M Wood, P W Jones
}

Thorax 2006;61:284-289. doi: 10.1136/thx.2005.051029

See end of article for authors' affiliations

Correspondence to

Correspondence to:
Dr E H Baker, Cardiac and Vascular Sciences

(Respiratory), St George's, University of London, Cranmer Terrace, London SW17 ORE, UK; ebaker@ sgul.ac.uk

Received 4 August 2005 Accepted 4 January 2006 Published Online First 31 January 2006 poor outcomes from pneumonia, myocardial infarction Background: Hyperglycaemia is associated with poor outcomes from pneumonia, myocardial infarction
and stroke, but the effect of blood glucose on outcomes from acute exacerbations of chronic obstructive pulmonary disease (AECOPD) has not been established. Recent UK guidelines do not comment on measurement or control of blood glucose in AECOPD. A study was therefore undertaken to determine the relationship between blood glucose concentrations, length of stay in hospital, and mortality in patients admitted with AECOPD.

Methods: Data were retrieved from electronic records for patients admitted with AECOPD with lower respiratory tract infection in 2001-2. The patients were grouped according to blood glucose quartile (group 1, <6 mmol// $(\mathrm{n}=69)$; group 2, 6.0-6.9 mmol// $(\mathrm{n}=69) ;$ group 3, 7.0-8.9 mmol// $(\mathrm{n}=75)$; and group 4, $>9.0 \mathrm{mmol} / \mathrm{l}(\mathrm{n}=71))$.

Results: The relative risk (RR) of death or long inpatient stay was significantly increased in group 3 (RR 1.46, $95 \% \mathrm{Cl} 1.05$ to $2.02, \mathrm{p}=0.02$ ) and group 4 (RR $1.97,95 \% \mathrm{Cl} 1.33$ to $2.92, \mathrm{p}<0.0001$ ) compared with group 1. For each $1 \mathrm{mmol} / \mathrm{l}$ increase in blood glucose the absolute risk of adverse outcomes increased by $15 \%(95 \% \mathrm{Cl} 4$ to 27), $p=0.006$. The risk of adverse outcomes increased with increasing hyperglycaemia independent of age, sex, a previous diagnosis of diabetes, and COPD severity. Isolation of multiple pathogens and Staphylococcus aureus from sputum also increased with increasing blood glucose.

Conclusion: Increasing blood glucose concentrations are associated with adverse clinical outcomes in patients with AECOPD. Tight control of blood glucose reduces mortality in patients in intensive care or following myocardial infarction. A prospective study is now required to determine whether control of blood glucose can also improve outcomes from AECOPD.
A pproximately 1.5 million people in the United Kingdom suffer from chronic obstructive pulmonary disease (COPD), ${ }^{1}$ and exacerbations of this condition have a major impact on personal and public health. Acute exacerbations of COPD (AECOPD) contribute to more than 100000 hospital admissions, one million inpatient bed days, and 30000 deaths annually in England and Wales. ${ }^{2}$ In this context, strategies to reduce mortality and length of stay from AECOPD are urgently required. Previous studies have shown that in-hospital mortality from AECOPD is predicted largely by fixed factors such as older age, ${ }^{3}$ male sex, co-morbidity and higher income, ${ }^{4}$ but also by arterial pH. ${ }^{3}$ It is not known whether hyperglycaemia, which is remediable, predicts outcomes of hospitalisation for AECOPD.

Hyperglycaemia is of interest as it is associated with poor outcomes from acute hospital admission for other conditions. In a study of 2030 adults admitted to general hospital wards, newly discovered hyperglycaemia (admission or fasting blood glucose $>7 \mathrm{mmol} / \mathrm{l}$ or two random blood glucose measurements $>11.1 \mathrm{mmol} / \mathrm{l}$ ) was associated with higher in-hospital mortality $(16 \%)$ than established diabetes mellitus $(3 \%)$ or normal blood glucose (1.7\%). ${ }^{5}$ Furthermore, hospital stay was longer and admission to the intensive care unit (ICU) was more frequent in those with new hyperglycaemia. In 2471 patients with community acquired pneumonia, those with blood glucose levels of $>11 \mathrm{mmol} / \mathrm{l}$ on admission had an increased risk of death and in-hospital complications compared with those with blood glucose levels $\leqslant 11 \mathrm{mmol} /$ 1. ${ }^{6}$ The risk of in-hospital complications increased by $3 \%$ for each $1 \mathrm{mmol} / \mathrm{l}$ increase in blood glucose. Hyperglycaemia has also been associated with adverse outcomes from acute myocardial infarction, ${ }^{7}$ ischaemic or haemorrhagic stroke, ${ }^{8}$ surgery, ${ }^{9}$ and trauma. ${ }^{10}$

Diabetes mellitus and acute hyperglycaemia are common in people with COPD. In the general population women with COPD had an 1.8-fold increased risk of developing type 2 diabetes compared with those without COPD. ${ }^{11}$ A previous diagnosis of diabetes mellitus was recorded in 14-15\% of patients admitted to hospital with AECOPD. ${ }^{12}{ }^{13}$ Treatment of AECOPD with oral glucocorticoids was associated with an increased risk of developing hyperglycaemia (odds ratio (OR) $5.48,95 \%$ confidence interval (CI) 1.58 to 18.96$).{ }^{14}$ Patients with AECOPD and diabetes treated with insulin had a longer in-patient stay and more frequent isolation of Gram negative bacteria from sputum than those without diabetes. ${ }^{14}$ Hyperglycaemia $(>11 \mathrm{mmol} / \mathrm{l})$ on admission predicted failure of non-invasive ventilation and infectious pulmonary complications in patients admitted to the ICU with acute respiratory failure caused by severe AECOPD. ${ }^{15}$

Hyperglycaemia is thus associated with a poor outcome from a wide range of acute illnesses including community

Abbreviations: $A E C O P D$, acute exacerbation of chronic obstructive pulmonary disease; $\mathrm{FEV}_{1}$, forced expiratory volume in 1 second; $\mathrm{FVC}$, forced vital capacity; ICU, intensive care unit 
acquired pneumonia, and is common in patients with AECOPD. However, the relationship between blood glucose levels and clinical outcomes in AECOPD has not been fully established. Furthermore, recently published national UK guidelines ${ }^{2}$ do not comment on whether blood glucose should be measured or controlled in the management of AECOPD. We therefore performed a retrospective pilot study to determine the relationship between blood glucose concentrations and clinical outcomes in patients admitted with AECOPD. The rationale for the retrospective design was to establish whether hyperglycaemia was associated with poor outcomes from AECOPD before investing in a large scale prospective study to determine whether tight control of blood glucose could improve the prognosis of AECOPD.

\section{METHODS}

\section{Participants}

ICD-10 codes were used to identify patients admitted to St George's Hospital in 2001 and 2002 with a discharge diagnosis of "acute exacerbation of COPD with lower respiratory tract infection" (code J44.0). The single admission for patients admitted once and the first admission for those admitted two or more times were included in the study. Information was obtained for each person using electronic patient records. This was not considered by the local research ethics committee to require ethical approval.

\section{Demographic data}

The age and sex of the study participants were noted. Lung function records were searched to retrieve forced expiratory volume in 1 second $\left(\mathrm{FEV}_{1}\right)$, forced vital capacity (FVC), and $\mathrm{FEV}_{\mathrm{l}} / \mathrm{FVC} \%$ from up to 2 years before or after hospital admission. These spirometric measurements were used to confirm the diagnosis and determine the severity of COPD using GOLD criteria. ${ }^{16}$

\section{Blood glucose levels}

Blood glucose measurements taken on admission and during the hospital stay were obtained for each participant from electronic patient records where available. Where more than one blood glucose measurement was made for an individual, the highest value was included in the analysis. Participants were divided into four groups by blood glucose quartiles. ICD-10 codes E10-14 were used to identify people with a discharge diagnosis of diabetes mellitus.

\section{Clinical outcomes}

Admission and discharge dates or dates of death (if death occurred in hospital) were retrieved and used to calculate length of stay and in-hospital mortality. A composite adverse outcome was defined as death or length of stay longer than the median length of stay for analysis.

\section{Sputum culture}

Sputum microbiology results were retrieved for each individual where available and the bacteria isolated were recorded.

\section{Analysis of data}

Normally distributed values are given as mean (SD) and were compared between two groups using unpaired $t$ tests and between more than two groups using analysis of variance. Non-normally distributed values are expressed as median (interquartile range, IQR) and were compared between two groups using the Mann-Whitney $U$ test and between more than two groups using the Kruskal-Wallis test. Categorical results are given as absolute numbers and percentages and compared between groups using $\chi^{2}$ analysis. Logistic regression was used to estimate the change in risk of outcomes per $1 \mathrm{mmol} / \mathrm{l}$ change in blood glucose, adjusted for age, sex,

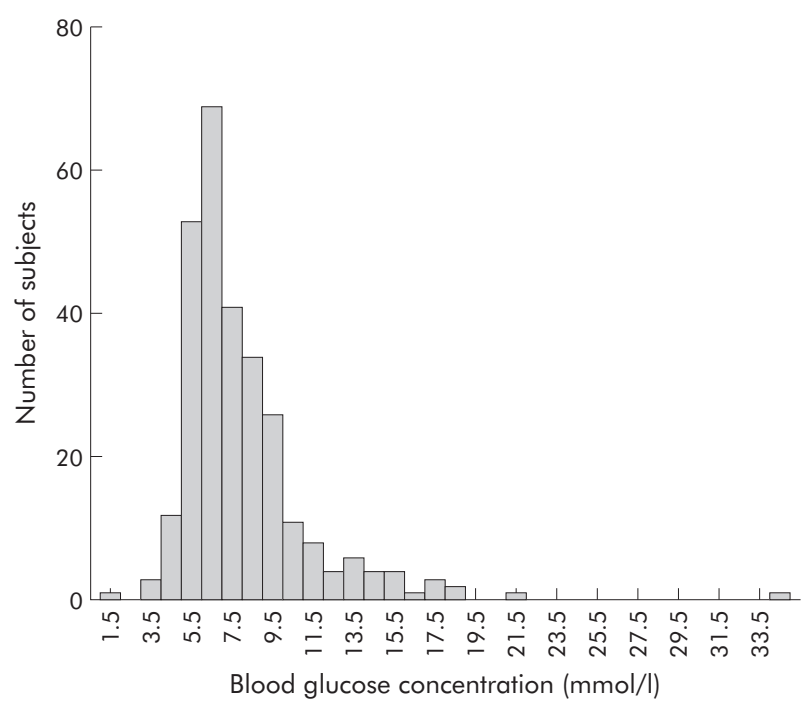

Figure 1 Histogram showing the highest blood glucose concentration recorded during inpatient stay for each of the 284 participants with an acute exacerbation of COPD.

previous diagnosis of diabetes mellitus, and $\mathrm{FEV}_{1} \%$ predicted. $p$ values of $<0.05$ were considered significant. Software used for the analysis was Statistical Package for the Social Sciences Version 11.5.

\section{RESULTS}

\section{Demographic data}

A total of 433 admissions with "acute exacerbation of COPD with lower respiratory tract infection" encoded J44.0 were identified. A single admission for 291 subjects admitted once and the first admission for 57 admitted two or more times were included in the analysis $(n=348 ; 195(56 \%)$ men, mean (SD) age 74.4 (10.4) years). Spirometric data were available for 119 of the 348 subjects (34\%), of whom $105(88 \%)$ met the criteria for a diagnosis of GOLD stage l COPD or worse.

\section{Blood glucose measurements}

Blood glucose was measured at admission for 252 of the 348 participants $(72 \%)$ and at or during admission for 284 (82\%; fig 1). Where more than one blood glucose measurement was made for an individual, the highest value was included in the analysis. In 193 participants $(68 \%)$ the admission blood glucose measurement was used for analysis. The blood glucose level was $>6.1 \mathrm{mmol} / \mathrm{l}$ in 204 participants (72\%) and $>11.1 \mathrm{mmol} / \mathrm{l}$ in $32(11 \%)$. The median blood glucose concentration was $7.0 \mathrm{mmol} / \mathrm{l}$ (IQR 6.0-9.0). Participants were divided into four groups by blood glucose quartiles: group 1, <6.0 mmol/l; group 2, 6.0-6.9 mmol/l; group 3, 7.0$8.9 \mathrm{mmol} / \mathrm{l}$; and group $4,>9.0 \mathrm{mmol} / \mathrm{l}$ (table 1 ). Fifteen of 284 participants $(5.3 \%$ ) had a diagnosis of diabetes recorded on their discharge summary.

\section{Relationship between blood glucose concentrations and adverse outcomes \\ Composite adverse outcomes}

The median length of stay was 9 (IQR 5-17) days. 154 participants $(44 \%)$ were judged to have had a good clinical outcome from their AECOPD (survival and length of stay $\leqslant 9$ days) while 194 (56\%) were considered to have had an adverse clinical outcome (death or length of stay $>9$ days).

The proportion of participants in each blood glucose quartile who had an adverse clinical outcome is shown in table 1 . The relative risk of an adverse outcome was 1.30 (95\% CI 0.93 to 1.82 ) in group 2, 1.46 (95\% CI 1.05 to 2.02 ) in group 3, and 1.97 (95\% CI 1.33 to 2.92 ) in group 4 compared 
Table 1 Demographic data, clinical outcomes, and sputum culture results in study participants grouped according to blood glucose quartiles

\begin{tabular}{|c|c|c|c|c|c|}
\hline & $\begin{array}{l}\text { Group } 1 \\
(n=69)\end{array}$ & $\begin{array}{l}\text { Group } 2 \\
(n=69)\end{array}$ & $\begin{array}{l}\text { Group } 3 \\
(n=75)\end{array}$ & $\begin{array}{l}\text { Group } 4 \\
(\mathrm{n}=71)\end{array}$ & $\mathrm{p}$ value \\
\hline \multicolumn{6}{|l|}{ Demographic data } \\
\hline Blood glucose $(\mathrm{mmol} / \mathrm{l})$ & $<6.0$ & $6.0-6.9$ & $7.0-8.9$ & $>9.0$ & \\
\hline Mean (SD) age (years) & $73.1(10.7)$ & $72.9(11.1)$ & $76.7(8.6)$ & $75.0(10.5)$ & 0.087 \\
\hline $\operatorname{Sex}(M: F)$ & $43: 26$ & $39: 30$ & $43: 32$ & $42: 29$ & 0.743 \\
\hline Pre-existing diagnosis of diabetes, $\mathrm{n}(\%)$ & $1(1.4 \%)$ & $1(1.4 \%)$ & $3(4 \%)$ & $10(14.1 \%)$ & 0.002 \\
\hline \multicolumn{6}{|l|}{ Clinical outcomes } \\
\hline Composite adverse outcomes, $\mathrm{n}(\%)$ & $28(41 \%)$ & $37(54 \%)$ & $45(60 \%)$ & $51(72 \%)$ & 0.0001 \\
\hline Mortality, n (\%) & $8(12 \%)$ & $11(16 \%)$ & $16(21 \%)$ & $22(31 \%)$ & 0.003 \\
\hline Median (IQR) length of stay (days) & $7(4-14)$ & $9(5-16)$ & $10(6-22)$ & $12(5-21)$ & 0.087 \\
\hline \multicolumn{6}{|l|}{ Sputum culture results } \\
\hline At least one pathogen, $\mathrm{n}(\%)$ & $14(56 \%)$ & $17(61 \%)$ & $19(66 \%)$ & $22(73 \%)$ & 0.154 \\
\hline Multiple pathogens, $\mathrm{n}(\%)$ & $3(12 \%)$ & $5(18 \%)$ & $8(28 \%)$ & $10(33 \%)$ & 0.031 \\
\hline Staphylococcus aureus, n (\%) & $1(4 \%)$ & $3(11 \%)$ & $8(28 \%)$ & $8(27 \%)$ & 0.011 \\
\hline Yeasts, n (\%) & $0(0 \%)$ & $3(11 \%)$ & $2(7 \%)$ & $5(17 \%)$ & 0.065 \\
\hline
\end{tabular}

Logistic regression was used for analysis of univariate relationships between glucose (divided by quartiles into four exposure levels) and categorial variables. Analysis of variance was used to compare age between the four groups and Kruskal-Wallis testing used to compare length of stay (not normally distributed) between the groups.

with participants in group 1 (lowest blood glucose quartile). The absolute risk of an adverse outcome increased by $15 \%$ (95\% CI 4 to 27 ) per $1 \mathrm{mmol} / \mathrm{l}$ increase in blood glucose $(\mathrm{p}=0.006)$ after adjustment for age, sex, and previous diagnosis of diabetes mellitus.

In the subgroup of 193 participants whose admission blood glucose measurements were used for analysis, the relative risk of an adverse outcome was 1.69 (95\% CI 1.08 to 2.64 ) in group 2, 1.53 (95\% CI 1.01 to 2.34 ) in group 3, and 1.98 (95\% CI 1.33 to 2.96 ) in group 4 compared with participants in group 1. In this subgroup, in whom admission blood glucose was used for analysis, the absolute risk of adverse outcomes increased by $31 \%$ (95\% CI $11-55)$ per $1 \mathrm{mmol} / \mathrm{l}$ increase in blood glucose $(\mathrm{p}=0.002)$ after adjustment for age, sex, and previous diagnosis of diabetes mellitus.

\section{Mortality}

The proportion of participants in each group who died is shown in table 1 . The mean (SD) blood glucose concentration was $7.7(2.8) \mathrm{mmol} / \mathrm{l}$ in patients who survived $(\mathrm{n}=227)$ and 9.1 (4.7) $\mathrm{mmol} / \mathrm{l}$ in those who died during the admission $(\mathrm{n}=57, \mathrm{p}=0.004)$. The relative risk of death was $1.22(95 \%$ CI 0.70 to 2.12) for patients in group 2, 2.10 (95\% CI 0.82 to 5.20 ) for those in group 3, and 3.42 (95\% CI 1.40 to 8.36 ) for those in group 4 compared with participants in group 1 . The risk of death increased by $10 \%$ (95\% CI $0-22$ ) per $1 \mathrm{mmol} / \mathrm{l}$ increase in blood glucose $(p=0.055)$ after adjustment for age, sex, and previous diagnosis of diabetes mellitus.

\section{Clinical outcomes in participants who did and did not have blood glucose measured}

Sixty four participants (18\%) did not have blood glucose measured during the admission. The outcomes in these were compared with those in participants who did have a blood glucose measurement to determine whether missing values may have confounded the results. The risk of an adverse outcome was similar in those who did not and those who did have glucose measured (52\% v 57\%, p=0.456). Mortality ( $11 \% v 20 \%, \mathrm{p}=0.088)$ and length of stay (9 (IQR 4-12) days $v 9$ (IQR 5-18) days, $\mathrm{p}=0.064)$ were slightly but not significantly lower in participants who did not have blood glucose measured.

\section{Clinical outcomes in participants who did and did} not have spirometric tests

Spirometric parameters were measured in 119 participants, 105 of whom (88\%) met the criteria for a diagnosis of GOLD stage 1 COPD or worse. ${ }^{16}$ Twenty four of these $(23 \%)$ had mild COPD (mean (SD) $\mathrm{FEV}_{1} 61$ (11)\% predicted), 53 (50\%) had moderate COPD ( $\mathrm{FEV}_{1} 38(6) \%$ predicted), and $28(27 \%)$ had severe COPD ( $\mathrm{FEV}_{1} 24$ (5)\% predicted). The spirometric findings were abnormal in the 14 participants who did not meet GOLD criteria for COPD (FEV 58 (19)\% predicted, $\mathrm{FEV}_{1} / \mathrm{FVC}$ ratio $\left.80(9) \%\right)$. In 88 participants with spirometric data who also had blood glucose measurements available, blood glucose quartiles were significantly related to an adverse outcome on univariate logistic regression (unadjusted OR 1.51 (95\% CI 1.02 to 2.23$)$ ). This relationship persisted after adjustment for age and sex (adjusted OR 1.70 (95\% CI 1.10 to 2.65)) and after further adjustment for FEV $\%$ predicted (adjusted OR 1.76 (95\% CI 1.12 to 2.76)). On multivariate analysis $\mathrm{FEV}_{1} \%$ predicted was not a significant determinant of adverse outcome (adjusted OR 1.02 (95\% CI 0.99 to 1.06$))$.

\section{Relationship between blood glucose concentrations and sputum culture results}

Sputum culture results were available for 112 participants $(32 \%)$ in whom blood glucose was measured at some point during the admission. There was no difference in the frequency of isolation of Streptococcus pneumoniae, Haemophilus influenzae, Moraxella catarrhalis, and Pseudomonas aeruginosa from the sputum between the glucose quartile groups. However, multiple pathogens and Staphylococcus aureus were isolated significantly more often in the sputum from participants in the higher blood glucose quartiles (table 1).

\section{DISCUSSION}

This study shows that increasing blood glucose concentrations are associated with adverse clinical outcomes in patients admitted to hospital with a physician diagnosis of AECOPD. Patients with higher blood glucose concentrations were more likely to die or to have a longer than median stay in hospital than those with lower blood glucose concentrations independent of age, sex, and a previous diagnosis of diabetes. In the subgroup of patients who had COPD confirmed by spirometric testing, the blood glucose quartile independently predicted adverse clinical outcomes whereas underlying COPD severity did not. Our results show that the association between acute hyperglycaemia, increased mortality, and a longer stay in hospital previously described for other conditions ${ }^{5-9} 1718$ is also seen in patients with AECOPD. Hyperglycaemia should therefore be added to the previously known risk factors for in-hospital mortality from 
AECOPD—namely, arterial $\mathrm{pH}^{3}{ }^{3}$ older age, ${ }^{3}{ }^{4}$ male sex, comorbidity, and higher income. ${ }^{4}$ Further studies are now required to determine whether control of blood glucose can influence outcomes from AECOPD.

In this study we determined the association between blood glucose and outcomes by dividing patients into quartiles determined by blood glucose concentrations. The risk of adverse outcomes was significantly increased in groups 3 and 4 (higher blood glucose) compared with group 1 (lowest blood glucose). This implies that random blood glucose at concentrations $\geqslant 7 \mathrm{mmol} / \mathrm{l}$ is detrimental in AECOPD and has implications when setting targets for blood glucose control. Studies of other conditions have also attempted to define blood glucose concentrations that determine clinical outcomes. Blood glucose concentrations of $>11.1 \mathrm{mmol} / \mathrm{l}$ were associated with a longer stay in hospital and increased mortality for general hospital admissions ${ }^{5}$ and increased mortality and in-hospital complications from community acquired pneumonia. ${ }^{6}$ Control of blood glucose levels to $\leqslant 11.0 \mathrm{mmol} / \mathrm{l}$ reduced mortality by $29 \%$ following myocardial infarction. ${ }^{19}$ Other studies have confirmed our findings that lower blood glucose concentrations $(6.0-11.1 \mathrm{mmol} / \mathrm{l})$ are associated with poor outcomes, including increased mortality and poor functional recovery from stroke ${ }^{8}$ and increased mortality and infection on a cardiothoracic intensive care unit (CICU). ${ }^{20}$ Tight control of blood glucose levels to $4.4-6.1 \mathrm{mmol} / \mathrm{l}$ in CICU patients reduced mortality, septicaemia, and prolonged antibiotic treatment compared with conventional treatment (blood glucose $10-11.1 \mathrm{mmol} / \mathrm{l}){ }^{20}$

\section{Potential mechanisms}

Our study shows an association between increased blood glucose concentrations and poor clinical outcomes in AECOPD, but does not explain this relationship. Blood glucose is increased in acute illness due to a combination of metabolic effects such as raised plasma catecholamine and glucocorticoid hormone concentrations and increased peripheral insulin resistance. ${ }^{21}$ Increased blood glucose concentrations in AECOPD could therefore simply be a marker for more severe illness which, in turn, results in adverse clinical outcomes. Blood glucose concentrations also rise with corticosteroid treatment for AECOPD, ${ }^{14}$ hence increased blood glucose concentrations could reflect administration of steroids at larger doses or for longer in people who are more unwell, who have poor clinical outcomes. However, treatment studies have shown that a reduction in blood glucose concentrations improves clinical outcomes, at least in patients in ICUs $^{20}$ or following myocardial infarction. ${ }^{19}$ Regression analysis suggested that control of blood glucose levels, rather than insulin dose, was responsible for the clinical benefits observed. ${ }^{22}$ These findings imply that increased glucose concentrations could have a direct detrimental effect on outcomes.

Hyperglycaemia could have adverse effects in acute illness through cellular glucose overload and oxidative stress. In acute illness, cytokines, hormones and hypoxia upregulate expression and membrane localisation of glucose transporters in many cell types. ${ }^{23}$ Cellular glucose overload results in increased glucose metabolism, in turn increasing superoxide and peroxynitrite production which may impair mitochondrial activity. ${ }^{23}$ In support of this, ultrastructural abnormalities were observed in hepatic mitochondria obtained at liver biopsy from ICU patients with hyperglycaemia, whereas virtually no mitochondrial abnormalities were detected in patients where normoglycaemia was maintained therapeutically. ${ }^{24}$ Mitochondrial toxicity of glucose in diverse cells could account for the broad spectrum of organ and tissue dysfunction associated with hyperglycaemia in acute illness. ${ }^{20}$
Hyperglycaemia could also cause adverse outcomes from AECOPD by predisposing to infection through systemic or local effects on host immunity or bacterial growth. In the present study we found that participants in higher blood glucose groups were more likely to have more than one type of organism or Staphylococcus aureus isolated from sputum than those in lower blood glucose groups. Systemic immune defects in people with diabetes are well documented and include decreased neutrophil and macrophage chemotaxis, phagocytosis, and killing and impairment in complement and cytokine responses to infection..$^{25}$ Our group has recently shown that local glucose concentrations in human airway secretions are normally extremely low. ${ }^{26}$ However, when blood glucose is raised above a threshold of $6.7-9.7 \mathrm{mmol} / \mathrm{l}$, glucose becomes detectable in airway secretions at concentrations of $1-11 \mathrm{mmol} / \mathrm{l} .^{26}{ }^{27}$ Patients intubated in ICUs who had glucose in bronchial aspirates were more likely than those without glucose in bronchial aspirates to have respiratory pathogens detected in aspirates, particularly methicillin resistant Staphylococcus aureus. ${ }^{28}$ Glucose in airway secretions could predispose to respiratory infection by promoting bacterial growth or interfering with local innate immunity.

\section{Strengths and weaknesses of study}

We studied the relationship between blood glucose concentration and clinical outcomes using a retrospective study. This had the advantage of allowing efficient collection and analysis of data from a large number of patients with AECOPD in whom the clinical outcome was already established. Our retrospective study had a number of limitations, some of which we were able to address during study design and analysis.

We identified people for inclusion in this study using the ICD-10 code J44.0 (acute exacerbation of COPD with lower respiratory tract infection). ICD-10 codes refer to the International Classification of Diseases, and disease codes are allocated by clinical clerks on the basis of summaries for hospital inpatients written by junior hospital doctors at the time of patient death or discharge. As this system is therefore imprecise, we chose to select patients with ICD-10 code J44.0. We felt that this code required a positive diagnosis of COPD exacerbation rather than other codes such as J44.1 (COPD with acute exacerbation unspecified) which were more likely to have been used as a default code. In this retrospective study we did not have the smoking histories of the participants to support the COPD diagnosis or chest radiographic results to exclude pneumonia. However, we were able to obtain spirometric test results for $34 \%$ of the participants which confirmed the diagnosis of GOLD stage 1 COPD or worse in $88 \%$ and was abnormal in the remaining $12 \%$. In this subgroup, ICD code J44.0 therefore did accurately identify subjects with COPD. It is not clear whether this implies that J44.0 was used accurately for the whole group irrespective of lung function, or that availability of spirometric results ensured a correct diagnosis of COPD. However, blood glucose was an independent predictor of poor clinical outcome in both the whole group with physician diagnosed AECOPD and in the subgroup with COPD confirmed by spirometry, which implies that our findings are applicable to both patient groups. Interestingly, in those with COPD confirmed by spirometry, hyperglycaemia was a stronger predictor of adverse outcomes from acute exacerbations than severity of COPD.

In this retrospective study we used random blood glucose measurements taken at admission $(68 \%)$ or during inpatient stay $(32 \%)$ to determine hyperglycaemia, and we were unable to standardise the timing of blood glucose measurements. Inhospital measurements may have differed from admission 
measurements-for example, corticosteroid treatment may increase blood glucose levels ${ }^{14}$ or resolution of the stress response to acute illness could lower blood glucose levels. ${ }^{21}$ Because of the retrospective study design, we were unable to explore the effect of these potential confounders on the relationship between blood glucose quartiles and clinical outcomes. However, we were able to confirm the relationship between blood glucose quartiles and adverse outcomes in the subgroup of patients whose admission blood glucose measurements were used for analysis, in whom measurement timing was more standardised. This subgroup analysis does not completely exclude the confounding effects of corticosteroids as we were not able to determine whether participants were taking steroids before admission. Further investigation of the important confounding effects of steroid treatment will require a prospective study.

An additional limitation of this study was the lack of information about the time spent by each individual within different bands of hyperglycaemia and this too requires prospective investigation.

Blood glucose measurements were obtained from electronic records and were not available for $18 \%$ of the study participants, reducing the numbers included in the analysis. Mortality and length of stay were slightly but not significantly lower in those who did not have a blood glucose measurement, suggesting that this group may not have had a blood glucose measurement because they were less unwell. Alternatively, blood glucose measurements may have been omitted because they are not required in the AECOPD guidelines $^{29}$ or they might have been performed by nearpatient testing which was not recorded electronically. The use of retrospective data also meant that sputum culture results were available for only $32 \%$ of participants which limited the analysis of the relationship between blood glucose levels and sputum culture results.

Previous studies have shown that in-hospital mortality from AECOPD is predicted largely by fixed factors such as older age, ${ }^{3}{ }^{4}$ male sex, co-morbidity, and higher income, ${ }^{4}$ as well as by arterial $\mathrm{pH}^{3}$ In this study we were able to show that the relationship between hyperglycaemia and poor outcome was independent of age, sex, underlying severity of COPD, and a previous diagnosis of diabetes mellitus. The use of ICD-10 codes may have underestimated the prevalence of diabetes mellitus as only $5.3 \%$ of our study participants (but 14-15\% of patients admitted to hospital with AECOPD in other studies ${ }^{12}{ }^{13}$ ) had diabetes. Because the data were collected retrospectively, we were also unable to include the effects of other important covariates such as $\mathrm{pH}^{3}$ and other co-morbidities ${ }^{4}$ in our model.

\section{Implications}

We have shown that the absolute risk of an adverse outcome (death or hospital stay longer than 9 days) following admission for AECOPD is significantly increased if random blood glucose is $\geqslant 7 \mathrm{mmol} / \mathrm{l}$. A prospective study is now required to determine whether clinical outcomes from AECOPD can be improved by tight control of blood glucose. Studies of the effect on outcomes of controlling blood glucose to physiological concentrations have to date been performed on ICUs where close monitoring can ensure effective blood glucose control without hypoglycaemia. Protocols now need to be developed for safe and effective blood glucose control to physiological concentrations on general hospital wards. Blood glucose control to $7.2 \mathrm{mmol} / \mathrm{l}$ in a heterogeneous population of critically ill adult patients reduced hospital mortality by $29 \%$ and ICU length of stay by $11 \% .^{30}$ If blood glucose control has a similar impact on patient outcomes from AECOPD, this could significantly reduce the one million inpatient bed days and 30000 deaths annually attributable to COPD.

\section{ACKNOWLEDGEMENTS}

The authors are grateful for the help of the medical records and lung function departments at St George's NHS Trust for assistance in retrieving patient records.

\section{Authors' affiliations \\ E H Baker, C H Janaway, B J Philips, A L Brennan, D L Baines,}

D M Wood, P W Jones, Glucose and Pulmonary Infection Group, St George's, University of London, London SW17 ORE, UK

No external funding was received for the execution of this study. Competing interests: none.

All the authors contributed to the conception and design, or acquisition of data, or analysis and interpretation of data; drafting the article or revising it critically for important intellectual content; and final approval of the version to be published.

\section{REFERENCES}

1 Soriano JB, Maier WC, Egger P, et al. Recent trends in physician diagnosed COPD in women and men in the UK. Thorax 2000;55:789-94.

2 National Collaborating Centre for Chronic Conditions. National clinical guidelines on management of chronic obstructive pulmonary disease in adults in primary and secondary care. Thorax 2004;59(Suppl I): 1-232.

3 Warren PM, Flenley DC, Millar JS, et al. Respiratory failure revisited: acute excacerbations of chronic bronchitis between 1961-68 and 1970-76. Lancet 1980;1:467-70.

4 Patil SP, Krishnan JA, Lechtzin N, et al. In-hospital mortality following acute exacerbations of chronic obstructive pulmonary disease. Arch Intern Med 2003; 163:1 180-6

5 Umpierrez GE, Isaacs SD, Bazargan $\mathrm{H}$, et al. Hyperglycemia: an independent marker of in-hospital mortality in patients with undiagnosed diabetes. J Clin Endocrinol Metab 2002;87:978-82.

6 McAlister FA, Majumdar SR, Blitz S, et al. The relation between hyperglycemia and outcomes in 2,471 patients admitted to the hospital with communityacquired pneumonia. Diabetes Care 2005;28:810-5.

7 Capes SE, Hunt D, Malmberg K, et al. Stress hyperglycaemia and increased risk of death after myocardial infarction in patients with and without diabetes: a systematic overview. Lancet 2000;355:773-8.

8 Capes SE, Hunt D, Malmberg K, et al. Stress hyperglycemia and prognosis of stroke in nondiabetic and diabetic patients: a systematic overview Stroke $2001 ; 32: 2426-32$

9 Hill Golden S, Peart-Vigilance C, Kao WH, et al. Perioperative glycemic control and the risk of infectious complications in a cohort of adults with diabetes. Diabetes Care 1999:22:1408-14.

10 Yendarumi S, Fulda GJ, Tinkoff GH. Admission hyperglycemia as a prognostic indicator in trauma. J Trauma 2003;55:33-8.

11 Rana JS, Mittleman MA, Sheikh J, et al. Chronic obstructive pulmonary disease, asthma and risk of type 2 diabetes in women. Diabetes Care 2004;27:2478-84.

12 Loukides S, Polyzogopoulos D. The effect of diabetes mellitus on the outcome of patients with chronic obstructive pulmonary disease exacerbated due to respiratory infections. Respiration 1996:63:170-3.

13 Antonelli IR, Fuso L, De Rosa M, et al. Co-morbidity contributes to predict mortality of patients with chronic obstructive pulmonary disease. Eur Respir $J$ 1997; 10:2794-800.

14 Wood-Baker RR, Gibson PG, Hannay M, et al. Systemic corticosteroids for acute exacerbations of chronic obstructive pulmonary disease. Cochrane Database Syst Rev 2005;(1):CD001288.

15 Moretti M, Cilione C, Tampieri A, et al. Incidence and causes of non-invasive mechanical ventilation failure after initial success. Thorax 2000;55:819-25.

16 Pauwels RA, Buist AS, Calverley PM, et al. Global strategy for the diagnosis, management and prevention of chronic obstructive pulmonary disease. NHLBI/WHO Global Initiative for Chronic Obstructive Lung Disease (GOLD) Workshop summary. Am J Respir Crit Care Med 2001;163:1256-76.

17 Pomposelli JJ, Baxter JK, Babineau TJ, et al. Early postoperative glucose control predicts nosocomial infection rate in diabetic patients. J Parenter Enteral Nutr 1998;22:77-81.

18 Yamashita S, Yamaguchi H, Sakaguchi M, et al. Longer-term diabetic patients have a more frequent incidence of nosocomial infections after elective gastrectomy. Anesth Analg 2000;91:1176-81.

19 Malmberg K, Ryden L, Efendic S, et al. Randomized trial of insulin-glucose infusion followed by subcutaneous insulin treatment in diabetic patients with acute myocardial infarction (DIGAMI study): effects on mortality at 1 year. J Am Coll Cardiol 1995;26:57-65.

20 Van den Berghe G, Wouters P, Weekers F, et al. Intensive insulin therapy in the critically ill patient. N Engl J Med 2001;345:1359-67.

21 Robinson LE, van Soeren MH. Insulin resistance and hyperglycemia in critical illness: role of insulin in glycemic control. AACN Clin Issues 2004; 15:45-62.

22 Finney SJ, Zekveld C, Elia A, et al. Glucose control and mortality in critically ill patients. JAMA 2003;290:2041-7. 
23 Van den Berghe G. How does blood glucose control with insulin save lives in intensive care? J Clin Invest 2004; 1 14:1187-95.

24 Vanhorebeek I, De Vos R, Mesotten D, et al. Protection of hepatocyte mitochondrial ultrastructure and function by strict blood glucose control with insulin in critically ill patients. Lancet 2005;365:53-9.

25 Geerlings SE, Hoepelman Al. Immune dysfunction in patients with diabetes mellitus (DM). FEMS. Immunol Med Microbiol 1999;26:259-65.

26 Philips BJ, Meguer J-X, Redman J, et al. Factors determining the appearance of glucose in upper and lower respiratory tract secretions. Intensive Care Med 2003;29:2204-10.
27 Wood DM, Brennan AL, Philips BJ, et al. Effect of hyperglycaemia on glucose concentration of airways secretions. Clin Sci 2004; 106:527-33.

28 Philips BJ, Redman J, Brennan AL, et al. Glucose in bronchial aspirates increases the risk of respiratory MRSA in intubated patients. Thorax 2005;60:761-4.

29 British Thoracic Society. BTS guidelines for the management of chronic obstructive pulmonary disease. Thorax 1997;52(Suppl 5): 1-28.

30 Krinsley J, Grissler B. Intensive glycemic management in critically ill patients. Jt Comm J Qual Patient Saf 2005;31:308-12.

\section{LUNG ALERT}

Can acetylcysteine slow lung function decline in idiopathic pulmonary fibrosis?

$\Delta$ Demedts M, Behr J, Buhl R, et al and the IFIGENIA Study Group. High-dose acetylcysteine in idiopathic pulmonary fibrosis. N Engl J Med 2005;353:2229-42

- fficient treatments in idiopathic pulmonary fibrosis (IPF) are elusive and patients die

$\mathrm{E}$ 3-5 years after diagnosis. The standard treatment for IPF includes prednisolone and azathioprine, but there is little evidence that these drugs alter the progress of the disease. This study suggests that the addition of high dose acetylcysteine to standard treatment with prednisone and azathioprine can slow the rate of lung function deterioration in patients with IPF.

155 patients were randomly assigned to a daily regimen of $1800 \mathrm{mg}$ acetylcysteine or a placebo, with both groups receiving prednisone and azathioprine. The patients had a diagnosis of usual interstitial pneumonia confirmed by high resolution computed tomography and histological findings. At 1 year the rate of loss of carbon monoxide transfer factor and vital capacity was slower in the group receiving acetylcysteine than in the placebo group, with a relative difference of $24 \%$ for transfer factor and $9 \%$ for vital capacity. There was no difference in patients' symptoms and the study was not powered to show a difference in survival.

Interestingly, patients taking acetylcysteine had a lower rate of myelotoxicity than those taking placebo. This prompted Hunninghake to ask in the accompanying editorial if "the effects of acetylcysteine are not better explained by the drug's prevention of the toxic effects of prednisone and azathioprine". Further studies are needed to determine whether acetylcysteine alone is an option for patients with IPF. This study suggests it should currently be considered as a treatment option in addition to immunosuppressive therapy.

T Toma

Clinical Fellow, Homerton University Hospital, London, UK; Homa@doctors.org.uk 